Supporting Information for

\title{
Structural Color Fibers Directly Drawn from Colloidal Suspensions with Controllable Optical Properties
}

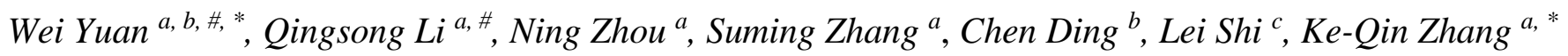

${ }^{a}$ National Engineering Laboratory for Modern Silk, College of Textile and Clothing Engineering, Soochow University, Suzhou 215123, China.

${ }^{b}$ Printable Electronics Research Centre, Suzhou Institute of Nano-tech and Nano-bionics, Chinese Academy of Sciences, Suzhou 215123, China.

${ }^{c}$ Department of Physics, Key Laboratory of Micro and Nano Photonic Structures (MOE) and Key Laboratory of Surface Physics, Fudan University, Shanghai 200433, China.

${ }^{\#} \mathrm{~W}$. Yuan and Q. Li contributed equally to this work.

\section{Corresponding Author}

*E-mail: kqzhang@suda.edu.cn. (K.-Q. Zhang)

*E-mail: wyuan2014@sinano.ac.cn. (W. Yuan) 


\section{Experimental section}

\section{Materials}

Styrene (St), methyl methacrylate (MMA), acrylic acid (AA), potassium persulfate (KPS), ammonium persulfate (APS), sodium dodecyl sulfonate (SDS), sodium dodecyl benzenesulfonate (SDBS), n-butyl acrylate (BA), ethanol, concentrated sulfuric acid (98\%), and hydrogen peroxide (30\%) were purchased from Shanghai Chemical Reagent Co. Ltd. (China) and used without further purification. Carbon black (99.5\%, $30 \mathrm{~nm})$ was bought from Aladdin, Co., Shanghai, China. The nanosilica (30 wt \%) was obtained from Eka Chemicals (Suzhou). The glass and PET fibers were provided by Jiangsu Hengtong Guangxian Technology Co., Ltd. and Jiangsu Hengli Chemical Fiber Co., Ltd. respectively.

\section{Synthesis of Polystyrene (PS) nanospheres.}

PS nanospheres were synthesized by emulsion polymerization in the absence of an emulsifier as we previously reported. ${ }^{\mathrm{s}}$ Briefly, Deionized water, ethanol, St and SDS were proportionally and sequentially added to a three-necked flask equipped a reflux condenser under mechanical stirring. Then APS was rapidly added after heating at $70{ }^{\circ} \mathrm{C}$ for $30 \mathrm{~min}$ under a nitrogen atmosphere. The reaction was allowed to proceed with continuous stirring at $80^{\circ} \mathrm{C}$ for $10 \mathrm{~h}$. The size of the PS nanospheres was tuned by varying the amount of SDS. The SEM images and size distribution of the synthesized PS nanospheres are shown in Figure S1.

\section{Preparation of $\mathbf{P}(\mathrm{St}-\mathrm{BA}-\mathrm{AA})$ nanospheres}

Monodispersed composite latex spheres of $\mathrm{P}(\mathrm{St}-\mathrm{BA}-\mathrm{AA})$ were synthesized by emulsion polymerization following reported procedures. ${ }^{\text {s2 }}$ Firstly, KPS (0.05 g) and SDBS (0-0.02 g) were dissolved in $120 \mathrm{~mL}$ DI water and added into a four-necked flask equipped with an $\mathrm{N}_{2}$ inlet and a reflux condenser. Then, St (5 g), BA (5 g) and AA (1 g) were added sequentially. After reaction at $75^{\circ} \mathrm{C}$ for $2 \mathrm{~h}$ under $\mathrm{N}_{2}$ atmosphere protection and $500 \mathrm{rpm}$ mechanical stirring, St (15 g), BA (15 g), AA ( $1 \mathrm{~g})$, and KPS (0.2 g) in water (15 g) were added drop by drop within 2 hours, and polymerization was further continued for $2 \mathrm{~h}$ with continuous stirring. The resulting latex 
suspension was used directly without further purification. The size distribution of P(St-BA-AA) nanospheres is shown in Figure S8.

\section{Fabrication of structural color fibers}

The concentration of PS nanosphere dispersions was controlled at the range of 1-5 wt $\%$. Carbon black (with a proportion of $1 \mathrm{wt} \%$ to PS) was added as short-wavelength absorbing additive. To increase the hydrophilicity, both glass and PET fibers were treated by oxygen plasma at $100 \mathrm{~W}$ for 3 min. A structural color fiber was prepared by directly drawing the treated fiber from the PS colloid suspensions with the assistance of an electric motor at a linear speed of $20-120 \mu \mathrm{m} / \mathrm{min}$. Finally, the composite fiber was dried at ambient conditions. For the preparation of non-crack structural color fiber, the mixture of $\mathrm{P}(\mathrm{St}-\mathrm{BA}-\mathrm{AA})$ nanospheres and nanosilica (with a proportion of $20 \mathrm{wt} \%$ to $\mathrm{P}(\mathrm{St}-\mathrm{BA}-\mathrm{AA}))$ were dispersed in DI water and the final concentration was fixed at $3 \mathrm{wt} \%$. Then $100 \mu \mathrm{m}$ pre-treated PET fiber was drawn from the suspension by the same procedure as aforementioned.

\section{Details on numerical calculation}

The simulations were conducted using a commercial software (DiffractMOD, RSoft Design Group) based on the rigorous coupled wave analysis (RCWA) method. To reduce the computation, the diameter of core fiber was fixed at $10 \mu \mathrm{m}$, PS spheres generated by MATLAB were closely packed onto the cylindrical fiber in an FCC structure. The refractive indexes of air and PS were taken as 1 and 1.6, respectively. The grid size is automatically selected by the software to be $\sim 5 \mathrm{~nm}$. To calculate the fibers with different layer numbers of PS spheres, the stacking layers perpendicular to the axes of fibers were tuned as desired. The angle-resolved reflectance spectra were collected by varying the simulated incident light. In order to get the simulated spectra of fibers with cracks on the surface, the PS spheres were randomly removed by lines (line defects) or by dots (point defects) at first, and then the calculation was conducted as normal.

\section{Characterization}


The morphologies of colloidal spheres, photonic crystal fibers were observed by a field emission scanning electron microscope (FE-SEM) (S-4800, Hitachi Ltd., Japan). The size of colloidal spheres was measured by dynamic light scattering (Zetasizer Nano S90, Malvern Instruments Ltd., England). Digital photos of fibers were taken by a Nikon DSLR camera (D5100). Microscopic pictures of colorful fibers were taken by a VHX-2000 digital microscope (Keyence Corporation, Japan). The surface morphologies of the fibers were measured by atomic force microscope (AFM) (Dimension Icon, Bruker Nano Inc., USA). The normal and angle-resolved reflectance spectra were collected by an angle-resolved microspectroscopy system (ARM160, Ideaoptics, PR China) as we previous reported (Figure S). ${ }^{\mathrm{s} 3}$

\section{Supplementary figures}
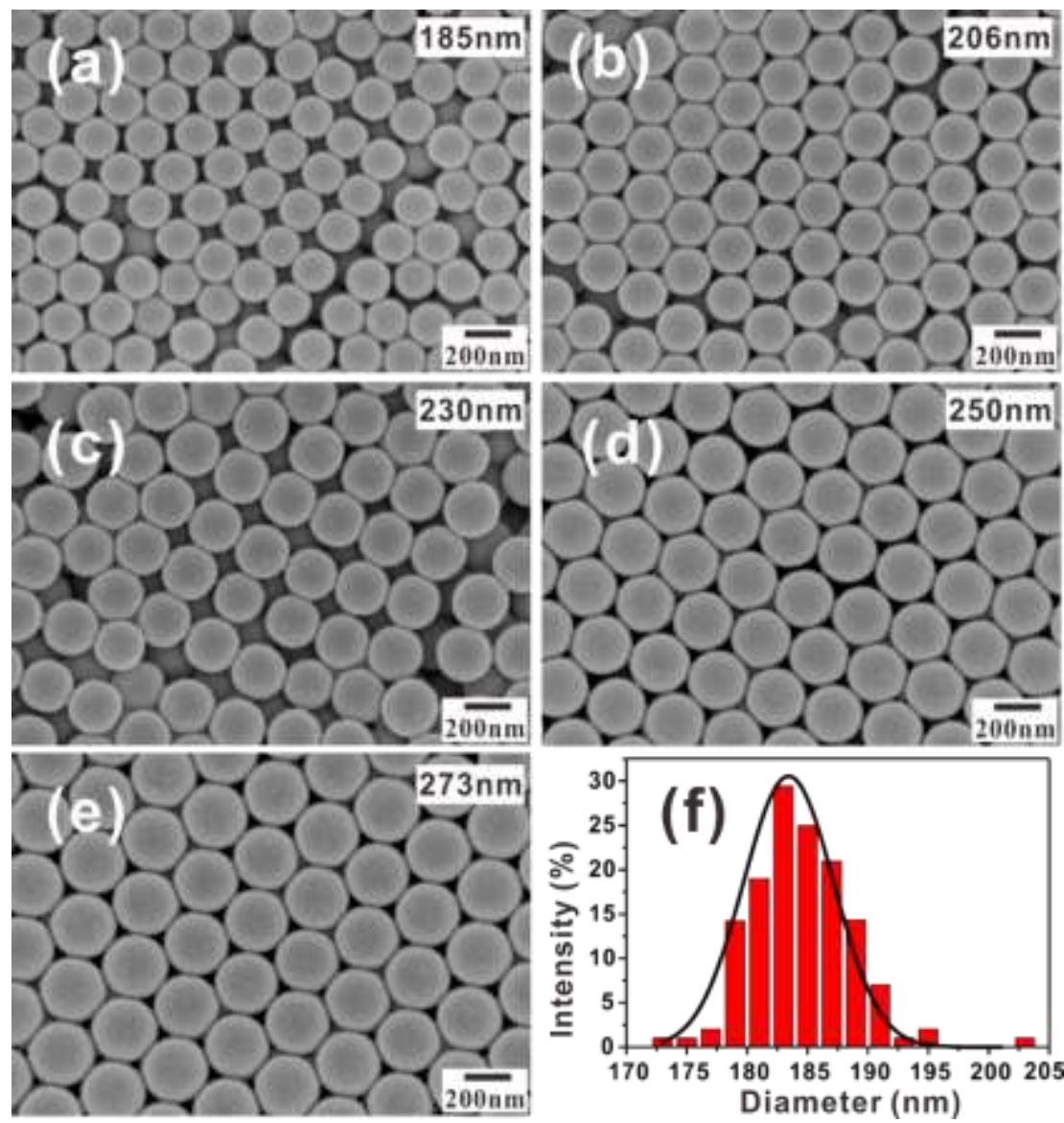

Figure S1. The SEM images of synthesized PS nanospheres with diameters of (a) 185 nm, (b) 206 $\mathrm{nm}$, (c) $230 \mathrm{~nm}$, (d) $250 \mathrm{~nm}$, and (e) $273 \mathrm{~nm}$. (f) The size distribution of $185 \mathrm{~nm}$ PS nanospheres 


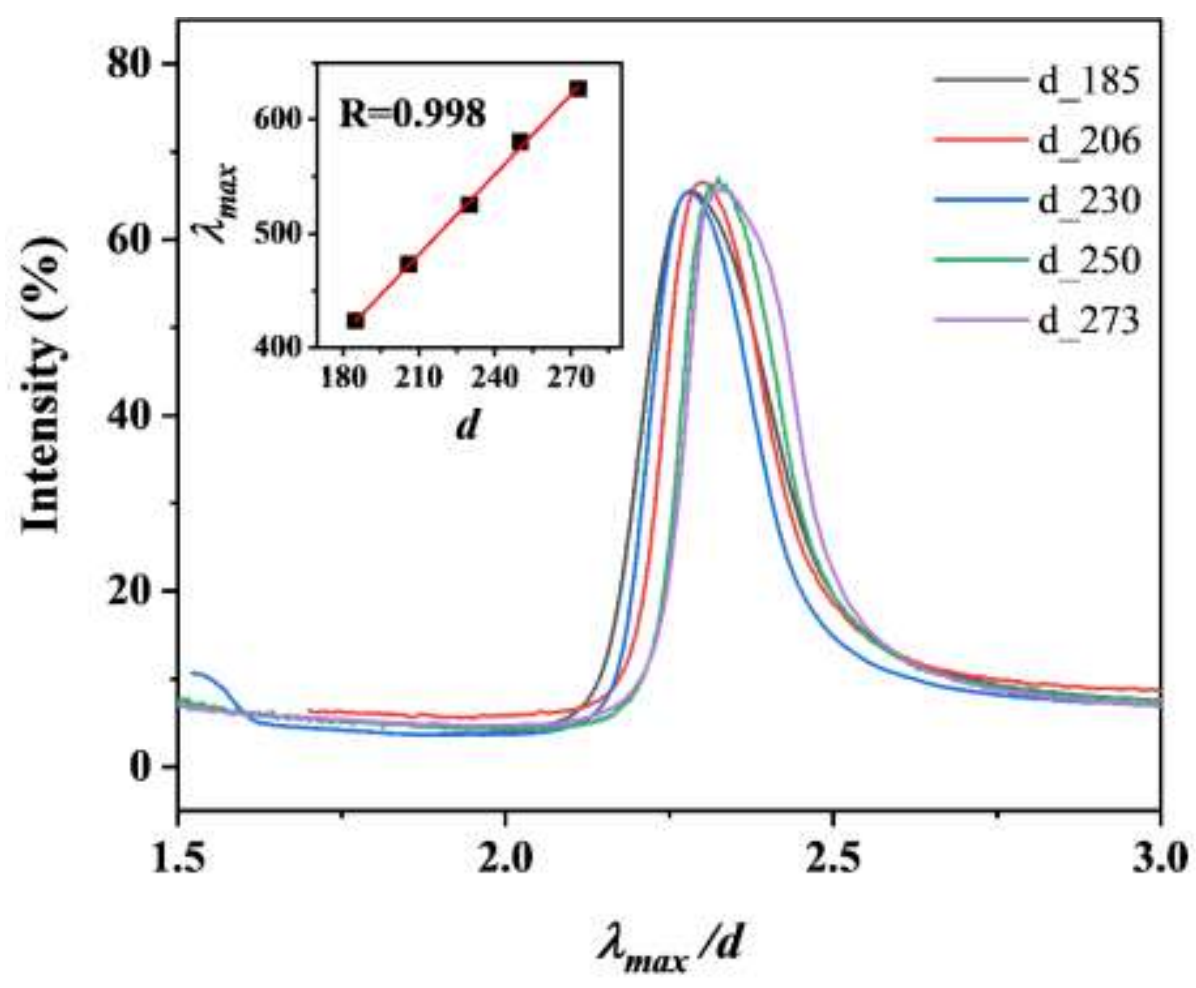

Figure S2. The reflectance spectra of 5 structural color fibers as a function of the ratios of peakwavelength/particle-size $\left(\lambda_{\max } / \boldsymbol{d}\right)$.

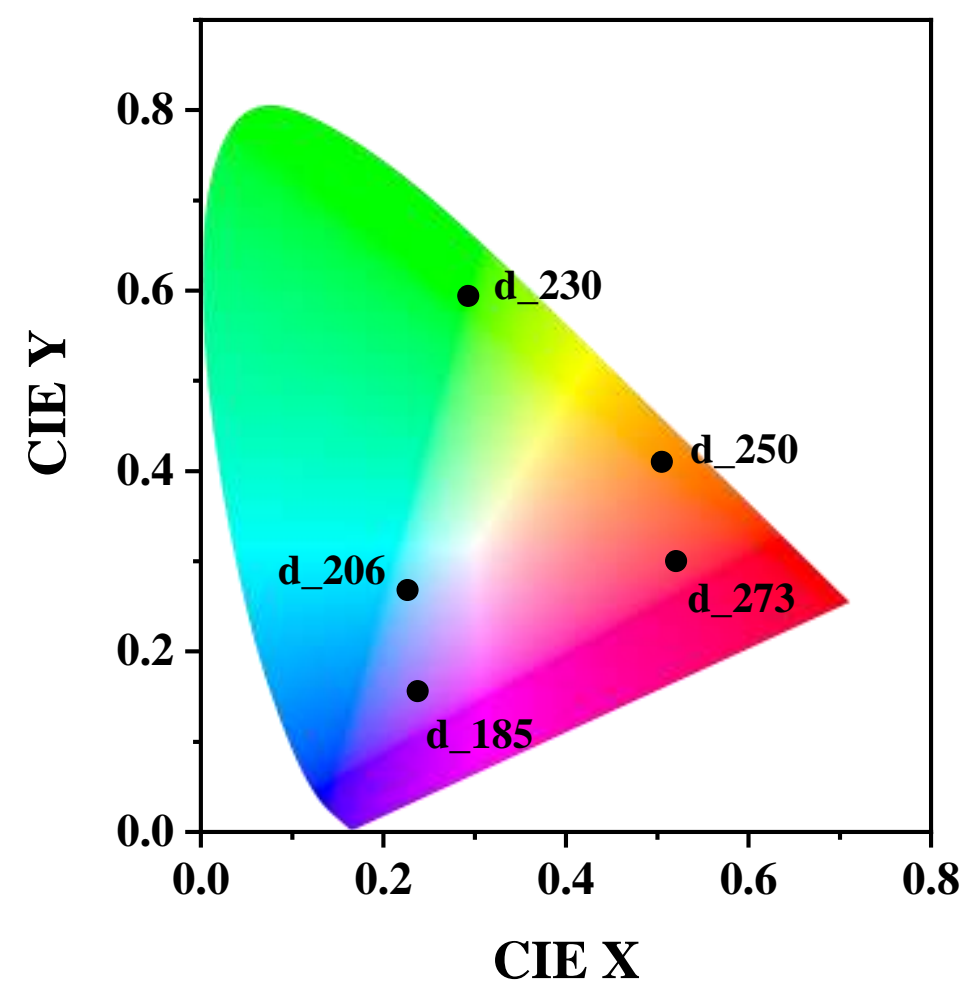

Figure S3. CIE chromaticity $\mathrm{x}$ and $\mathrm{y}$ values for the colors of the obtained 5 structural color fibers in Figure 2a 

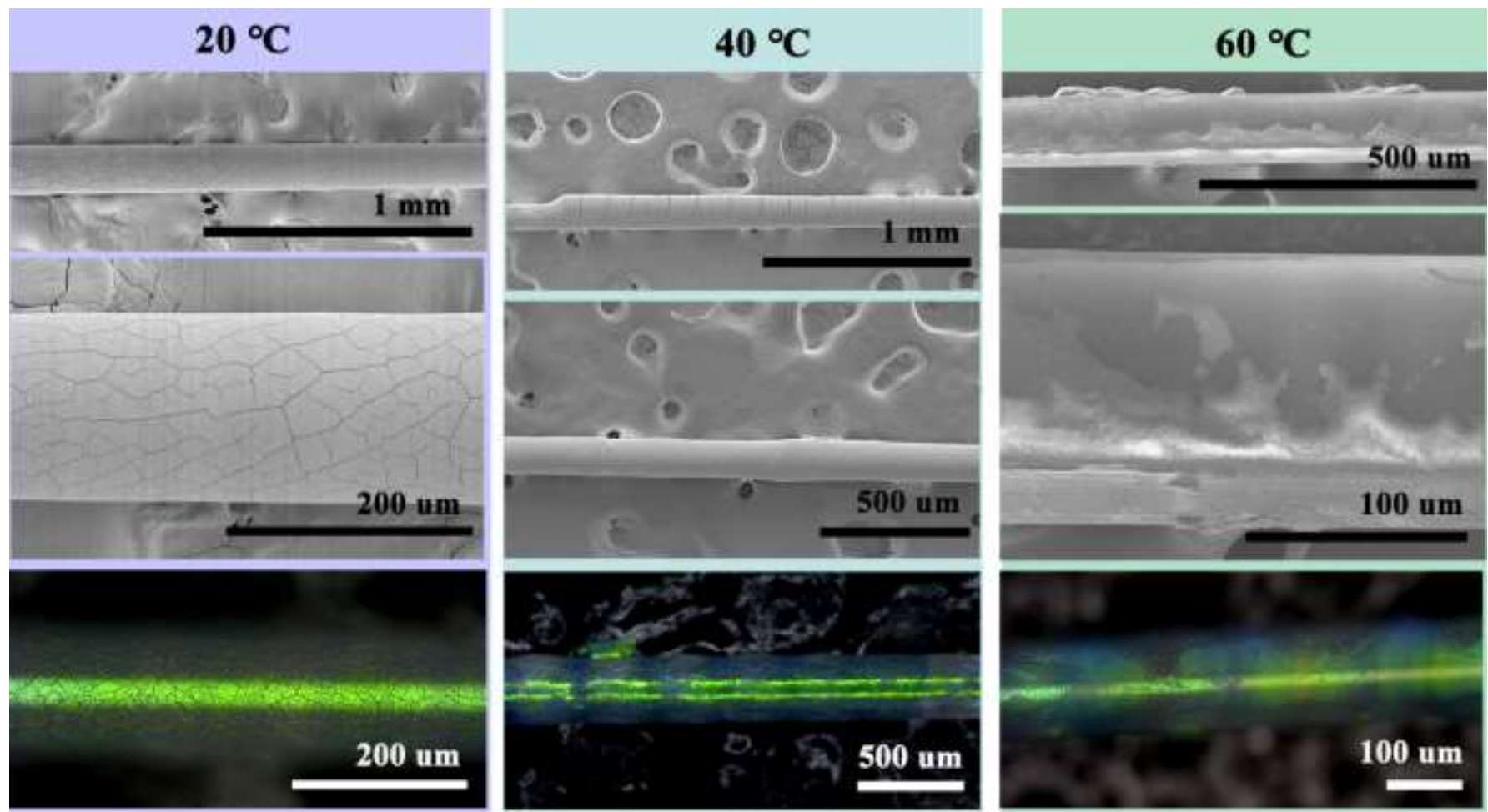

Figure S4. The SEM images and optical photos of structural color fibers using $125 \mu \mathrm{m}$ glass fibers and $230 \mathrm{~nm}$ PS nanospheres under different temperatures $\left(20^{\circ} \mathrm{C}, 40{ }^{\circ} \mathrm{C}\right.$, and $\left.60^{\circ} \mathrm{C}\right)$
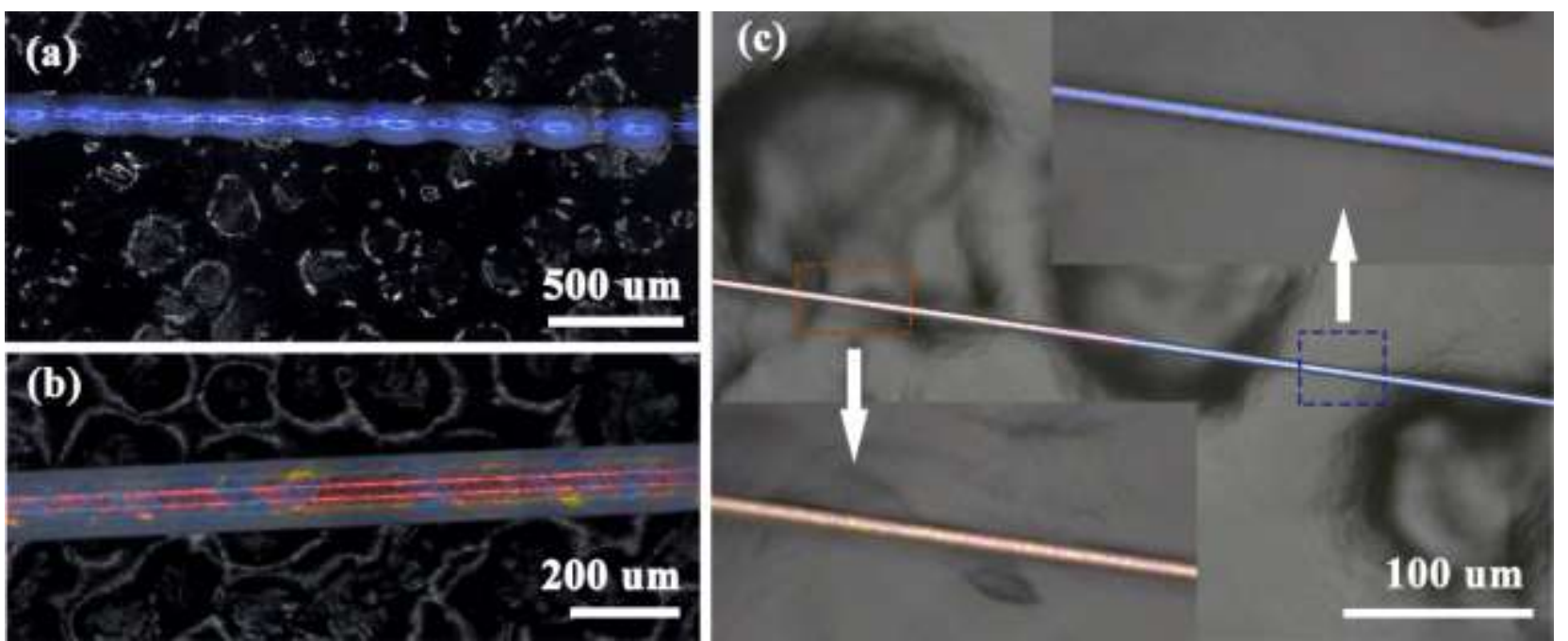

Figure S5. The optical images of structural color fibers with some special structures or unique optical effects. (a) "string beads" on a single glass fiber by using periodically varied drawing speeds. (b) Heterostructures on a glass fiber prepared by a two-step dip-coating process (206 nm PS spheres were coated at first, and $273 \mathrm{~nm}$ PS spheres were coated subsequently), the additive color mixing phenomenon can be observed. (c) Two colors on a single carbon fiber using a two-stage dip-coating process (206 nm PS spheres were coated on one part of the fiber, and then the coating solution as replaced by $250 \mathrm{~nm}$ PS spheres for the other part of the fiber) 


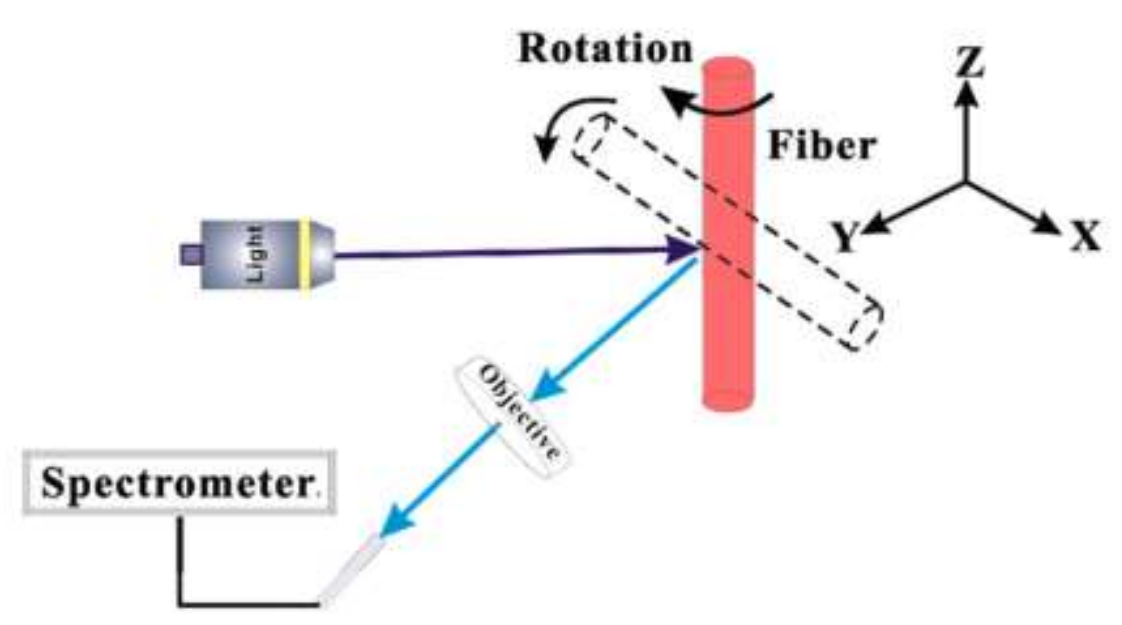

Figure S6. Schematic illustration for the measurement of angle-resolved reflectance spectra from direction along and perpendicular to the fibers. The picture is reprinted with permission from ref $\mathrm{s} 3$. Copyright 2013 American Chemical Society.
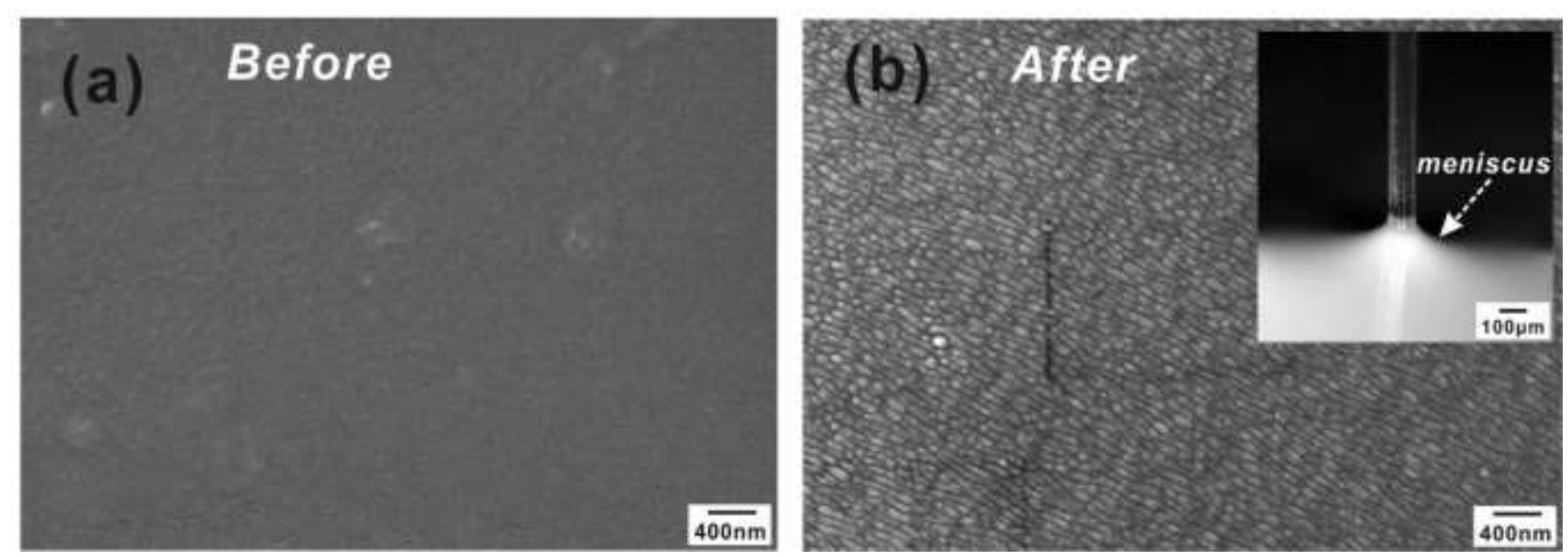

Figure S7. The SEM images of PET fibers (a) before and (b) after $\mathrm{O}_{2}$ plasma treatment. The inset in (b) shows the hydrophilicity of the treated PET fibers 


\section{Results}

$\begin{array}{rlllll} & & & \text { Size (d.n... } & \% \text { Intensity } & \text { Width (d.n... } \\ \text { Z-Average (d.nm): } 208.3 & \text { Peak 1: } & 216.4 & 100.0 & 45.55 \\ \text { Pdl: } 0.011 & \text { Peak 2: } & 0.000 & 0.0 & 0.000 \\ \text { Intercept: } 0.954 & \text { Peak 3: } & 0.000 & 0.0 & 0.000 \\ \text { Result quality } & & & & \end{array}$

\section{Size Distribution by Intensity}

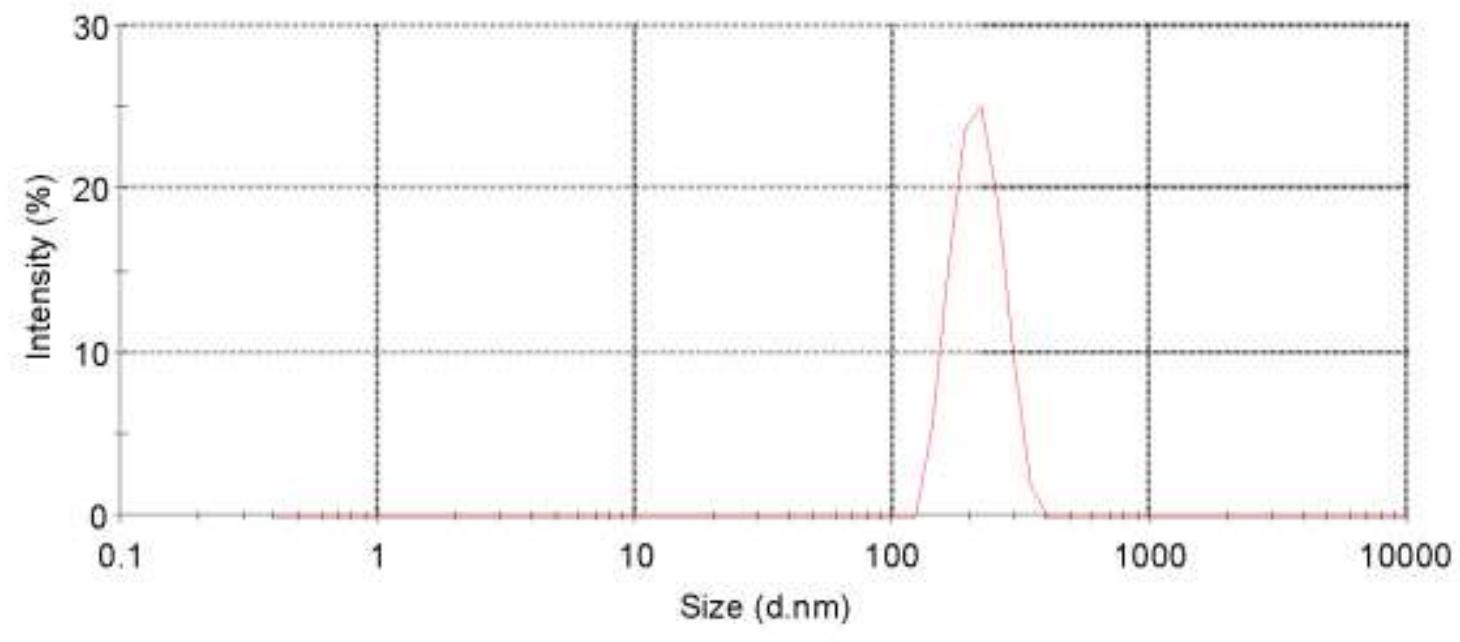

Figure S8. The screenshot of the measured size distribution of P(St-BA-AA) nanospheres by DLS
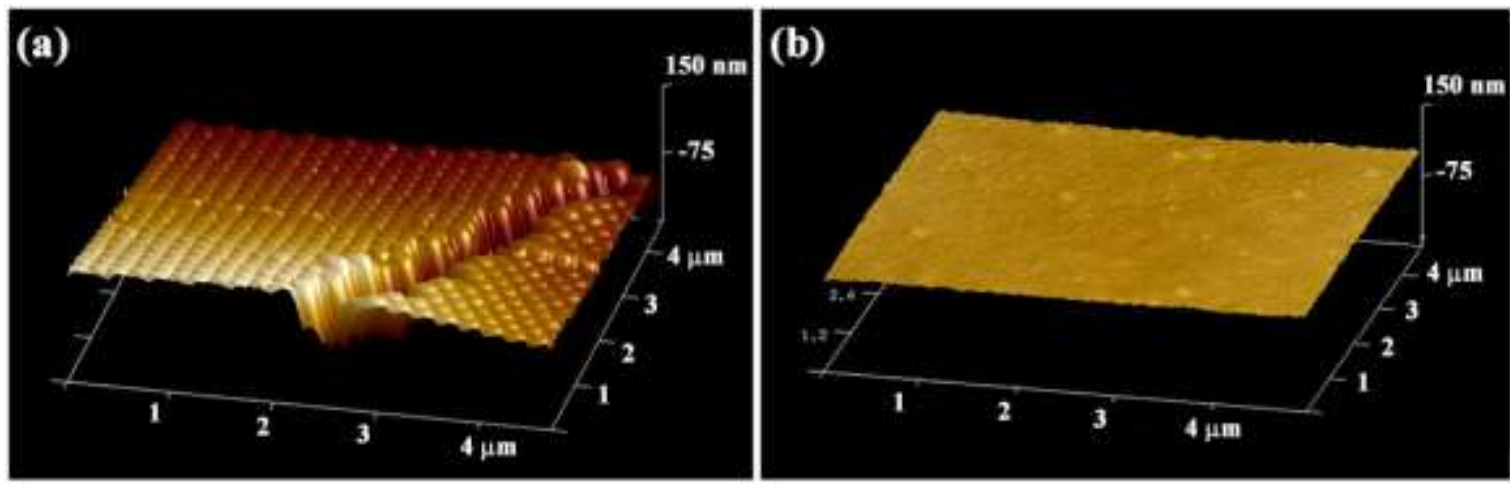

Figure S9. The AFM images of (a) PS nanospheres on glass fibers, and (b) P(St-BA-AA)/nanosilica on PET fibers 


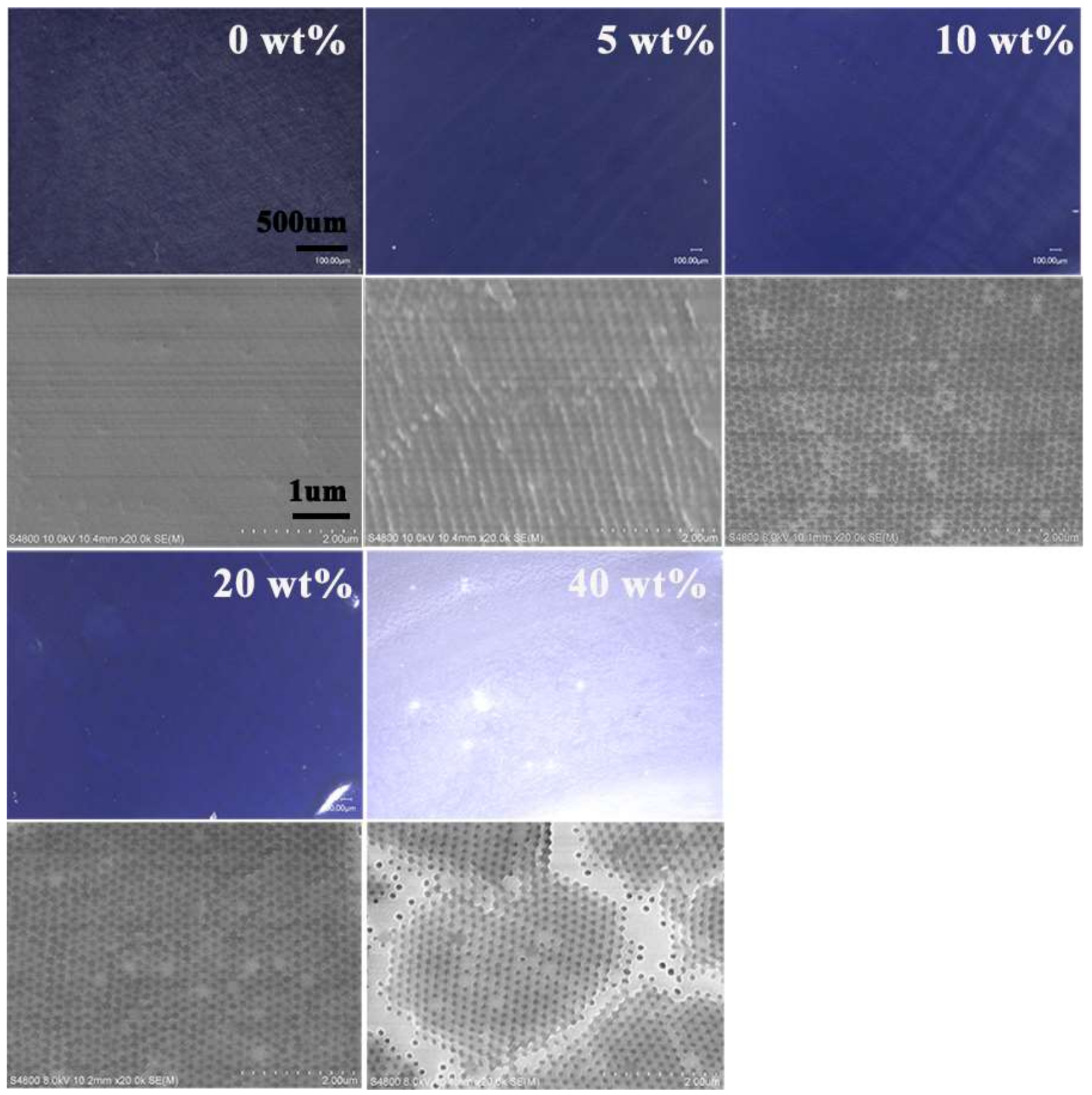

Figure S10. The optical photos and SEM images of P(St-BA-AA) spheres films with different nanosilica content on glass slides 


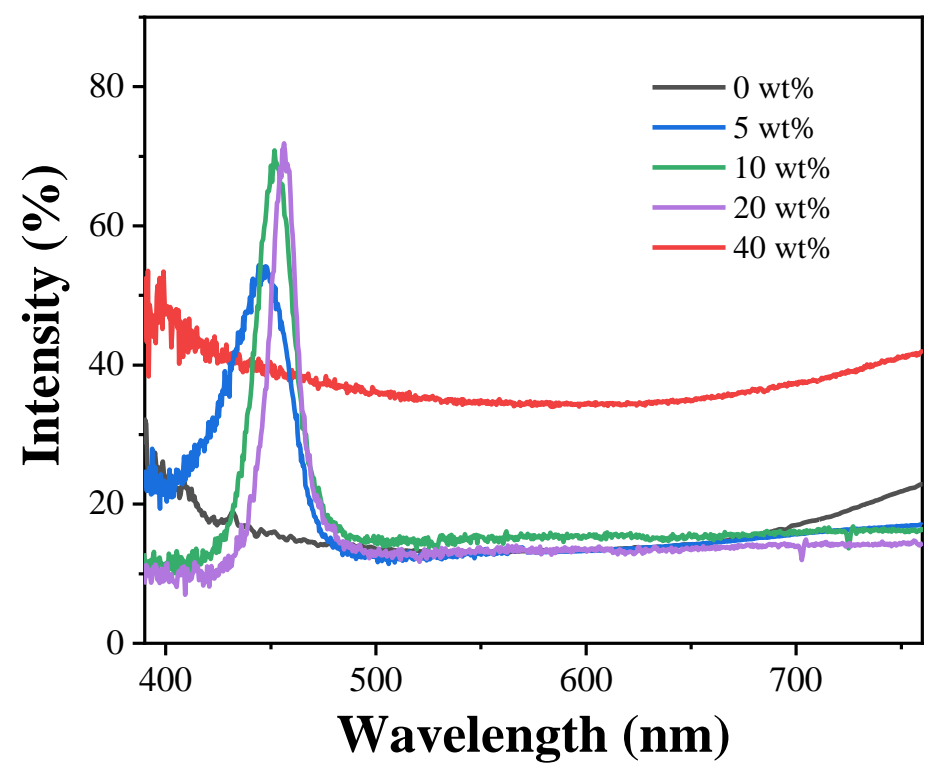

Figure S11. The reflectance spectra of P(St-BA-AA) spheres films with different nanosilica content on glass slides in Figure S9

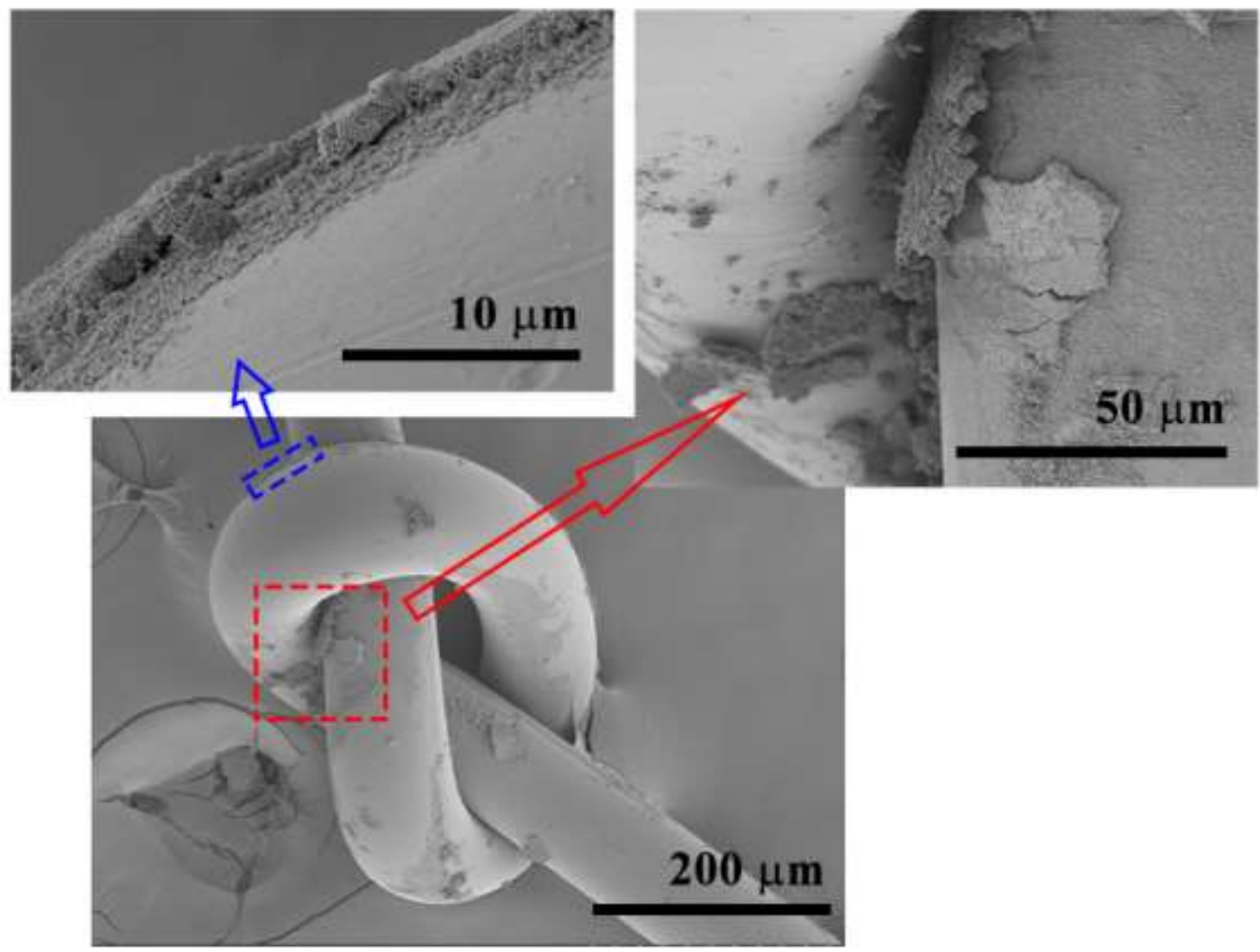

Figure S12. The SEM images of a knot made by PET fiber with PS colloid coating on the surface 


\section{References}

(S1) Yuan, W.; Zhang, K. Q. Structural Evolution of Electrospun Composite Fibers from the Blend of Polyvinyl Alcohol and Polymer Nanoparticles. Langmuir 2012, 28 (43), 15418-15424, DOI: 10.1021/la303312q.

(S2) You, B.; Wen, N. G.; Shi, L.; Wu, L. M.; Zi, J. Facile fabrication of a three-dimensional colloidal crystal film with large-area and robust mechanical properties. J. Mater. Chem. 2009, 19 (22), 3594-3597, DOI: 10.1039/b906293a.

(S3) Zhou, N.; Zhang, A.; Shi, L.; Zhang, K.-Q. Fabrication of Structurally-Colored Fibers with Axial Core-Shell Structure via Electrophoretic Deposition and Their Optical Properties. ACS Macro Letters 2013, 2 (2), 116-120, DOI: 10.1021/mz300517n. 